\title{
Hall Effect on Moving Isothermal Vertical Plat With Variable Temperature And Mass Diffusion In Thepresence of Rotating Fluid
}

\author{
R.MUTHUCUMARASWAMY', K.MUTHURACKU ALIAS PREMA \\ ${ }^{1}$ Department of Applied Mathematics, Sri Venkateswara College of Engineering \\ Pennalur, Sriperumbudur Taluk- 602117, India. \\ E-mail:msamy@svce.ac.in \\ ${ }^{2}$ Research Scholar \\ Department of Applied Mathematics, Sri Venkateswara College of Engineering \\ Pennalur, Sriperumbudur Taluk- 602117, India. \\ E-mail:kmprema66@yahoo.co.in
}

\begin{abstract}
The present work of this paper analyses with the effect of heat and mass transfer on free convection flow near an accelerated infinite vertical plate in the presence of transverse magnetic field. The surface is maintained at linear temperature and concentration variations and time dependent wall suction is assumed to occur at the permeable surface. The system of equations such as equation of momentum, energy, mass diffusion has been transformed by usual transformation into a non-dimensional form. Some applications of practical interest are discussed for deferent types of plate motions. The governing equations are solved by the use of Laplace transformation technique and the results obtained are presented graphically for (both primary and secondary) the velocity distribution for the influence of the various parameters like hall parameter, Hartmann number, rotation parameter, Schmidt number, Prandt number, thermal Grashof number, mass Grashof number. The temperature and concentration profiles are presented graphically.
\end{abstract}

Index Terms-Hall Effect, Isothermal, Temperature Diffusion, Mass Diffusion, Vertical Plate, Rotating fluid.

\section{INTRODUCTION}

The natural convection heat transfer from a vertical plate to a fluid has applications in many industrial processes. Practical applications are found in heat exchangers. The study of convection with heat-mass transfer is very useful in the fields as Chemistry, agriculture and oceanography. Heat and mass transfer from a vertical plate have been studied by several authors. An exact solution for the current is induced in the direction normal to both electric and magnetic fields. This phenomenon is known as the Hall Effect.

Aboeldahabn et al. [1] have analyzed Hall current effect on magneto hydrodynamic free convection flow past a semi infinite vertical plate with mass transfer. Acharya [2] studied Hall Effect with simultaneous thermal and mass diffusion on unsteady hydro magnetic flow near an accelerated vertical plate. Basanth Kumar Jha and Ravindra Prasad [3] presented free convection and mass transfer effects on the flow past an accelerated vertical plate with heat source. Chen [4] studied heat and mass transfer with variable wall temperature and concentration. Das et al. [5] studied radiation effects on flow past an impulsively started vertical infinite plate. Elbashbeshy [6] discussed heat and mass transfer along a vertical plate with variable surface tension and concentration in the presence of the magnetic field. Hetnarski [7] analyzed an algorithm for generating some inverse Laplace transforms of exponential form. Ganesh and Pilani [8] rendered Finite difference analysis of unsteady natural convection MHD past an inclined plate with variable surface heat and mass flux. Kafousias and Raptis [9]analyzed Mass transfer and free convection effects on the flow past an accelerated vertical infinite plate with variable suction or injection. Muthucumaraswamy [10] investigated the interaction of thermal radiation on vertical oscillating plate with variable temperature and mass diffusion. Raptisa et al. [11] studied hydro magnetic free convection flow past an accelerated vertical infinite plate with variable suction and heat flux. Sattar [12] rendered free convection and mass transfer flow through a porous medium past an infinite vertical porous plate with time dependent temperature and concentration. Takhar [13] investigated unsteady flow free convective flow over an infinite vertical porous plate due to the combined effects of thermal and mass diffusion, magnetic field and Hall current.

The objective of the present paper is to study the exact solution of Hall Effect on MHD flow past an accelerated infinite vertical plate with uniform mass diffusion, numerical methods and the developments in computer 
technology methods play an important role in solving the partial differential equations by Laplace-transform technique. The solutions are in terms of exponential and complementary error functions. The various parameters entering into the problem like hall parameter, rotation parameter, radiation parameter, Hartmann number, thermal Grashof number, mass Grashof number, Schmidt number, temperature are investigated and the results are shown with the help of their graphical representations.

\section{FORMULATION OF THE PROBLEM AND ITS SOLUTION}

The unsteady flow of an incompressible andelectrically conducting viscous fluid past an infinite vertical plate with variable temperature and variable mass diffusion has been considered. Relative to the rotating fluid, the plate is impulsively started from rest and set into motion with uniform acceleration in its own plane. When, the fluid and the plate rotate as a rigid body with a uniform angular velocity $\Omega^{\prime}$ about $\mathrm{z}$-axis in the presence of an imposed uniform transverse magnetic field $B_{0}$ normal to the plate. Also, no external electric field is applied, so the effect of polarization of the magnetic field is negligible. Initially, for time $t^{\prime} \leq 0$, the plate and fluid are at the same temperature $T_{\infty}^{\prime}$ and the mass concentration $C_{\infty}^{\prime}$. At time $t^{\prime}>0$, the plate temperature and the mass concentration is raised to $T_{w}^{\prime}$ and $C_{w}^{\prime}$. As the plate is infinitely long, the fluid velocity components and temperature distribution are functions of $\mathrm{z}$ and $\mathrm{t}$ only. Under the above assumptions as well as Boussinesq's approximation, the equations of conservation of Momentum, Energy and Concentration governing the free convection boundary layer flow past an exponentially accelerated vertical plate can be expressed as:

Equation of Momentum:

$$
\begin{aligned}
& \frac{\partial u}{\partial t^{\prime}}-2 \Omega^{\prime} v=\vartheta \frac{\partial^{2} u}{\partial z^{2}}-\frac{1}{\rho} \frac{\partial p}{\partial x}+g+\frac{B_{0}}{\rho} j_{y} \\
& \frac{\partial v}{\partial t^{\prime}}+2 \Omega^{\prime} u=\vartheta \frac{\partial^{2} v}{\partial z^{2}}-\frac{B_{0}}{\rho} j_{x}
\end{aligned}
$$

Equation of Energy:

$$
\rho C_{p} \frac{\partial T^{\prime}}{\partial t^{\prime}}=k \frac{\partial^{2} T^{\prime}}{\partial z^{2}}-\frac{\partial q_{r}}{\partial z}
$$

Equation of diffusion:

$$
\frac{\partial C^{\prime}}{\partial t^{\prime}}=D \frac{\partial^{2} C^{\prime}}{\partial z^{2}}
$$

term in Equation (1) vanishes for small and hence for the outer flow, beside there is no magnetic field along $\mathrm{x}$ direction gradient, so we have

$$
0=-\frac{\partial p}{\partial x}-\rho_{\infty} g
$$

By eliminating the pressure term from Equations (1) and (5), we obtain

$$
\frac{\partial u}{\partial t^{\prime}}-2 \Omega^{\prime} v=\vartheta \frac{\partial^{2} u}{\partial z^{2}}+\left(\rho_{\infty}-\rho\right) g+\frac{B_{0}}{\rho} j_{y}
$$

The Boussinesq approximation gives

$$
\rho_{\infty}-\rho=\rho_{\infty} \beta\left(T^{\prime}-T_{\infty}^{\prime}\right)+\rho_{\infty} \beta^{*}\left(C^{\prime}-C_{\infty}^{\prime}\right)
$$

On using (7) in the equation (6) and noting that $\rho_{\infty}$ is approximately equal to 1 , the momentum equation reduces to

$$
\begin{aligned}
& \frac{\partial u}{\partial t^{\prime}}-2 \Omega^{\prime} v=\vartheta \frac{\partial^{2} u}{\partial z^{2}}+\frac{B_{0}}{\rho} j_{y}+g \beta\left(T^{\prime}-T_{\infty}^{\prime}\right) \\
& +g \beta^{*}\left(C^{\prime}-C_{\infty}^{\prime}\right)
\end{aligned}
$$

The generalized Ohm's law, on taking Hall currents into account and neglecting ion-slip and thermo-electric effect, is

$$
\vec{j}+\frac{\omega_{e} \tau_{e}}{B_{0}}(\vec{j} \times \vec{B})=\sigma(\vec{E}+\vec{q} \times \vec{B})
$$

The equation (9) gives

$$
\begin{aligned}
& j_{x}-m j_{y}=\sigma v B_{0} \\
& j_{y}+m j_{x}=-\sigma u B_{0}
\end{aligned}
$$

where $m=\omega_{e} \tau_{e}$ is the Hall parameter. Solving (10) and (11) for $j_{x}$ and $j_{y}$, we have

$$
\begin{aligned}
& j_{x}=\frac{\sigma B_{0}}{1+m^{2}}(v-m u) \\
& j_{y}=\frac{\sigma B_{0}}{1+m^{2}}(u+m v)
\end{aligned}
$$

On the use of (12) and (13), the momentum equations (8) and (2) become

$$
\begin{aligned}
& \frac{\partial u}{\partial t^{\prime}}=\vartheta \frac{\partial^{2} u}{\partial z^{2}}+2 \Omega^{\prime} v-\frac{\sigma B_{0}^{2}(u+m v)}{\rho\left(1+m^{2}\right)} \\
& +g \beta\left(T^{\prime}-T_{\infty}^{\prime}\right)+g \beta^{*}\left(C^{\prime}-C_{\infty}^{\prime}\right) \\
& \frac{\partial v}{\partial t^{\prime}}=\vartheta \frac{\partial^{2} v}{\partial z^{2}}-2 \Omega^{\prime} u+\frac{\sigma B_{0}^{2}(m u-v)}{\rho\left(1+m^{2}\right)}
\end{aligned}
$$

The initial and boundary conditions are given by

Since there is no large velocity gradient here, the viscous 


$$
\begin{aligned}
& t^{\prime} \leq 0, u=0, v=0, T^{\prime}=T_{\infty}^{\prime}, C^{\prime}=C_{\infty}^{\prime} \quad \forall z \\
& t^{\prime}>0, u=u_{0} v=0, T^{\prime}=T_{\infty}^{\prime}+\left(T_{w}^{\prime}-T_{\infty}^{\prime}\right) A t^{\prime},
\end{aligned}
$$

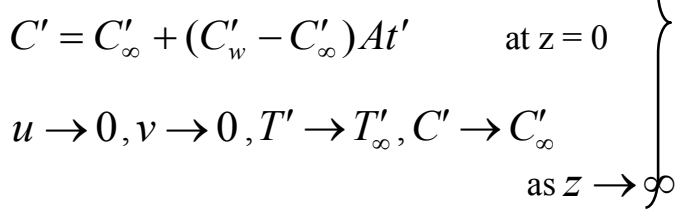

where $A=\frac{u_{0}^{2}}{\gamma}$

The local radiant for the case of an optically thin gray gas is expressed by

$$
\frac{\partial q_{r}}{\partial z}=-4 a^{*} \sigma\left(T_{\infty}^{\prime 4}-T^{\prime 4}\right)
$$

It is assumed that the temperature differences within the flow are sufficiently small such that $T^{\prime 4}$ may be expressed as a linear function of the temperature. This is accomplished by expanding $T^{\prime 4}$ in a Taylor series about $T_{\infty}^{\prime}$ and neglecting higher-order terms, thus

$$
T^{\prime 4} \cong 4 T_{\infty}^{\prime 3} T^{\prime}-3 T_{\infty}^{\prime 4}
$$

By using equations (17) and (18), equation (3) reduces to

$$
\rho C_{p} \frac{\partial T^{\prime}}{\partial t^{\prime}}=k \frac{\partial^{2} T^{\prime}}{\partial z^{2}}+16 a^{*} \sigma T_{\infty}^{\prime 3}\left(T_{\infty}^{\prime}-T^{\prime}\right)
$$

Let us introducing the following non-dimensional quantities

$$
\begin{aligned}
& U=\frac{u}{u_{0}}, V=\frac{v}{u_{0}}, Z=\frac{z u_{0}}{\gamma}, t=\frac{t^{\prime} u_{0}{ }^{2}}{\gamma}, \\
& \Omega=\frac{\Omega^{\prime} \gamma}{u_{0}{ }^{2}}, M^{2}=\frac{\Omega \sigma B_{0}{ }^{2} \gamma}{2 \rho u_{0}{ }^{2}}, \theta=\frac{T^{\prime}-T_{\infty}^{\prime}}{T_{w}^{\prime}-T_{\infty}^{\prime}}, \\
& C=\frac{C^{\prime}-C_{\infty}^{\prime}}{C^{\prime}{ }_{w}-C_{\infty}^{\prime}}, \operatorname{Pr}=\frac{\rho C_{p}}{k}, S c=\frac{v}{D}, \\
& G r=\frac{g \beta \gamma\left(T^{\prime}{ }_{w}-T_{\infty}^{\prime}\right)}{u_{0}{ }^{3}}, G c=\frac{g \beta^{*} \gamma\left(C^{\prime}{ }_{w}-C_{\infty}^{\prime}\right)}{u_{0}{ }^{3}}, \\
& R=\frac{16 a^{*} \sigma \gamma^{2} T^{\prime 3}{ }_{\infty}}{k u_{0}{ }^{2}}
\end{aligned}
$$

Using these boundary conditions in above equations, we obtain the following dimensionless form of the governing equations

$$
\frac{\partial U}{\partial t}=\frac{\partial^{2} U}{\partial Z^{2}}+2 \Omega V-\frac{2 M^{2}(U+m V)}{1+m^{2}}+G r \theta+G c C
$$

$$
\begin{aligned}
& \frac{\partial V}{\partial t}=\frac{\partial^{2} V}{\partial Z^{2}}-2 \Omega U+\frac{2 M^{2}(m U-V)}{1+m^{2}} \\
& \frac{\partial \theta}{\partial t}=\frac{1}{\operatorname{Pr}} \frac{\partial^{2} \theta}{\partial Z^{2}}-\frac{R}{\operatorname{Pr}} \theta \\
& \frac{\partial C}{\partial t}=\frac{1}{S c} \frac{\partial^{2} C}{\partial Z^{2}}
\end{aligned}
$$

The boundary conditions for corresponding order are

$$
\left.\begin{array}{c}
t \leq 0, U=0, V=0, \theta=0, C=0 \text { for all } Z \\
t>0, U=1, V=0, \theta=t, C=t \text { at } Z=0 \\
U \rightarrow 0, V \rightarrow 0, \theta \rightarrow 0 C \rightarrow 0 \text { as } Z \rightarrow \infty
\end{array}\right\}
$$

Now equations (20) \& (21) and boundary conditions (24) can be combined to give

$$
\frac{\partial F}{\partial t}=\frac{\partial^{2} F}{\partial Z^{2}}-a F+G r \theta+G c C
$$

Where $a=\frac{2 M^{2}}{1+m^{2}}+2 i\left(\Omega-\frac{M^{2} m}{1+m^{2}}\right)$

$$
\begin{aligned}
& \frac{\partial \theta}{\partial t}=\frac{1}{\operatorname{Pr}} \frac{\partial^{2} \theta}{\partial Z^{2}}-\frac{R}{\operatorname{Pr}} \theta \\
& \frac{\partial C}{\partial t}=\frac{1}{S c} \frac{\partial^{2} C}{\partial Z^{2}}
\end{aligned}
$$

The initial and boundary conditions in non-dimensional quantities are

$$
\left.\begin{array}{r}
\begin{array}{r}
t \leq 0, F=0, \theta=0, C=0 \text { for all } Z \\
t>0, F
\end{array}=1, \theta=t, C=t \text { at } Z=0 \\
F \rightarrow 0, \theta \rightarrow 0 C \rightarrow 0 \text { as } Z \rightarrow \infty
\end{array}\right\}
$$

Exact solution for the fluid temperature and concentration of (26), (27) is expressed in the following form by taking inverse Laplace transform of solution as

$$
\begin{gathered}
C(Z, t)=(t)\left(\begin{array}{l}
\left(1+2 \eta^{2} S c\right) \operatorname{erfc}(\eta \sqrt{S c}) \\
-\frac{2 \eta \sqrt{S c}}{\sqrt{\pi}} \exp \left(-\eta^{2} S c\right)
\end{array}\right) \\
\theta(Z, t)=\frac{t}{2}\left(\begin{array}{l}
\exp (2 \eta \sqrt{b \operatorname{Pr} t}) \operatorname{erf} c(\eta \sqrt{\operatorname{Pr}}+\sqrt{b t}) \\
+\exp (-2 \eta \sqrt{b \operatorname{Pr} t}) \operatorname{erf} c(\eta \sqrt{\operatorname{Pr}}-\sqrt{b t})
\end{array}\right)
\end{gathered}
$$




$$
-\frac{\eta \sqrt{\operatorname{Pr}} \sqrt{t}}{2 \sqrt{b}}\left(\begin{array}{l}
\exp (-2 \eta \sqrt{b \operatorname{Pr} t}) \operatorname{erfc}(\eta \sqrt{\operatorname{Pr}}-\sqrt{b t}) \\
-\exp (2 \eta \sqrt{b \operatorname{Pr} t}) \operatorname{erfc}(\eta \sqrt{\operatorname{Pr}}+\sqrt{b t})
\end{array}\right)
$$

The equations (25), (26), (27), subject to the boundary conditions (28), are solved by the usual Laplace-transform technique and the solutions are derived as follows:

$$
\begin{aligned}
& F(Z, t)=\left(1+a_{1}-a_{2}\right)\left(\frac{1}{2}\right)(\exp (2 \eta \sqrt{a t}) \\
& \operatorname{erfc}(\eta+\sqrt{a t})+\exp (-2 \eta \sqrt{a t}) \operatorname{erfc}(\eta-\sqrt{a t})) \\
& +a_{1} d\left[\left(\frac{t}{2}\right)(\exp (2 \eta \sqrt{a t}) \operatorname{erfc}(\eta+\sqrt{a t})\right. \\
& +\exp (-2 \eta \sqrt{a t}) \operatorname{erfc}(\eta-\sqrt{a t}))- \\
& \left(\frac{\eta \sqrt{t}}{2 \sqrt{a}}\right)(\exp (-2 \eta \sqrt{a t}) \operatorname{erfc}(\eta-\sqrt{a t}) \\
& -\exp (2 \eta \sqrt{a t}) \operatorname{erf} c(\eta+\sqrt{a t}))] \\
& -a_{2} e\left[\left(\frac{t}{2}\right)(\exp (2 \eta \sqrt{a t}) \operatorname{erfc}(\eta+\sqrt{a t})\right. \\
& +\exp (-2 \eta \sqrt{a t}) \operatorname{erfc}(\eta-\sqrt{a t}))- \\
& \left(\frac{\eta \sqrt{t}}{2 \sqrt{a}}\right)(\exp (-2 \eta \sqrt{a t}) \operatorname{erfc}(\eta-\sqrt{a t}) \\
& -\exp (2 \eta \sqrt{a t}) \operatorname{erfc}(\eta+\sqrt{a t}))] \\
& +a_{1} g(\exp (2 \eta \sqrt{(a+d) t}) \operatorname{erfc}(\eta+\sqrt{(a+d) t} \\
& +\exp (-2 \eta \sqrt{(a+d) t}) \operatorname{erfc}(\eta-\sqrt{(a+d) t})) \\
& -a_{2} h(\exp (2 \eta \sqrt{(a+e) t}) \operatorname{erfc}(\eta+\sqrt{(a+e) t}) \\
& +\exp (-2 \eta \sqrt{(a+e) t}) \operatorname{erf} c(\eta-\sqrt{(a+e) t})) \\
& -\frac{a_{1}}{2}(\exp (2 \eta \sqrt{\operatorname{Pr} b t}) \operatorname{erf} c(\eta \sqrt{\operatorname{Pr}}+\sqrt{b t}) \\
& +\exp (-2 \eta \sqrt{\operatorname{Pr} b t}) \operatorname{erf} c(\eta \sqrt{\operatorname{Pr}}-\sqrt{b t})) \\
& -a_{1} d\left[\left(\frac{t}{2}\right)(\exp (2 \eta \sqrt{\operatorname{Pr} b t}) \operatorname{erfc}(\eta \sqrt{\operatorname{Pr}}+\sqrt{b t})\right. \\
& +\exp (-2 \eta \sqrt{\operatorname{Pr} b t}) \operatorname{erfc}(\eta \sqrt{\operatorname{Pr}}-\sqrt{b t}))- \\
& \left(\frac{\eta \sqrt{\operatorname{Pr}} \sqrt{t}}{2 \sqrt{b}}\right)(\exp (-2 \eta \sqrt{\operatorname{Pr} b t}) \operatorname{erfc}(\eta \sqrt{\operatorname{Pr}}-\sqrt{b t}) \\
& -\exp (2 \eta \sqrt{\operatorname{Pr} b t}) \operatorname{erfc}(\eta \sqrt{\operatorname{Pr}}+\sqrt{b t})] \\
& +a_{1} g(\exp (2 \eta \sqrt{\operatorname{Pr}(b+d) t}) \operatorname{erfc}(\eta \sqrt{\operatorname{Pr}}+\sqrt{(b+d) t}) \\
& +\exp (-2 \eta \sqrt{\operatorname{Pr}(b+d) t}) \operatorname{erfc}(\eta \sqrt{\operatorname{Pr}}-\sqrt{(b+d) t}))
\end{aligned}
$$

$$
\begin{aligned}
& \quad+a_{2}(\operatorname{erfc}(\eta \sqrt{S c})) \\
& +a_{2} \operatorname{et}\left[\left(1+2 \eta^{2} S c\right) \operatorname{erf} c(\eta \sqrt{S c})-\frac{2 \eta \sqrt{S c}}{\sqrt{\pi}} \exp \left(-\eta^{2} S c\right)\right. \\
& \quad-a_{2} h(\exp (2 \eta \sqrt{S c e t}) \operatorname{erf} c(\eta \sqrt{S c}+\sqrt{e t}) \\
& +\exp (-2 \eta \sqrt{S c e t}) \operatorname{erfc}(\eta \sqrt{S c}-\sqrt{e t})) \\
& \text { where } \eta=\frac{z}{2 \sqrt{t}} ; a_{1}=\frac{G r}{(1-\operatorname{Pr}) d^{2}} ; a_{2}=\frac{G c}{(S c-1) e^{2}} ; \\
& b=\frac{R}{\operatorname{Pr}} ; d=\frac{b \operatorname{Pr}-a}{1-\operatorname{Pr}} ; e=\frac{a}{S c-1} ; g=\frac{\exp (d t)}{2} ; \\
& h=\frac{\exp (e t)}{2}
\end{aligned}
$$

In order to get the physical insight into the problem, the numerical values of $F$ have been computed from (31). While evaluating this expression, it is observed that the argument of the error function is complex and, hence, we have separated it into real and imaginary parts by using the following formula:

$$
\begin{aligned}
& \operatorname{erf}(a+i b)=\operatorname{erf}(a)+\frac{\exp \left(-a^{2}\right)}{2 a \pi}[1-\cos (2 a b)+i \sin (2 a b)] \\
& +\frac{2 \exp \left(-a^{2}\right)}{\pi} \sum_{n=1}^{\infty} \frac{\exp \left(-\frac{n^{2}}{4}\right)}{n^{2}+4 a^{2}}\left[f_{n}(a, b)+i g_{n}(a, b)\right]+\in(a, b)
\end{aligned}
$$

Where

$f_{n}=2 a-2 a \cosh (n b) \cos (2 a b)+n \sinh (n a b) \sin (2 a b)$ and

$$
\begin{gathered}
g_{n}=2 a \cosh (n b) \sin (2 a b)+n \sinh (n a b) \cos (2 a b) \\
|\in(a, b)| \approx 10^{-16}|\operatorname{erf}(a+i b)|
\end{gathered}
$$

\section{RESULTS AND DISCUSSION}

Results are computed for the dimensionless concentration $\mathrm{C}$, temperature $\theta$, the values of primary velocity $\mathrm{U}$, secondary velocity $\mathrm{V}$ are obtained for different values of the rotation parameter ' $\Omega$, radiation parameter $R$, Schmidt number Sc, Hartmann Number M. Hall parameter $\mathrm{m}$, thermal Grashof number Gr, mass Grashof number $\mathrm{Gc}$, and time $t$ and these numerical values are presented by means of different graphs. In order to understand the physical situation of the problem and hence the manifestations of the various material parameters entering the problem we have computed the numerical values using the software "Mat -Lab". The value of the Prandtl number Pr is chosen to represent air $(\mathrm{Pr}=0.71)$. The value of Schmidt number is chosen to represent water vapour $(\mathrm{Sc}=0.6)$ and keeping the values of time $t=0.2$. 


\section{A .CONCENTRATION PROFILE}

Figure 1 represents the effect of Schmidt number $(\mathrm{Sc}=0.3,0.6,0.78,2.01)$ on the concentration at time $\mathrm{t}=0.2$. The effect of concentration is important in concentration field. As Schmidt number (Sc) increases the concentration decreases while the frequency of excitation remains constant.

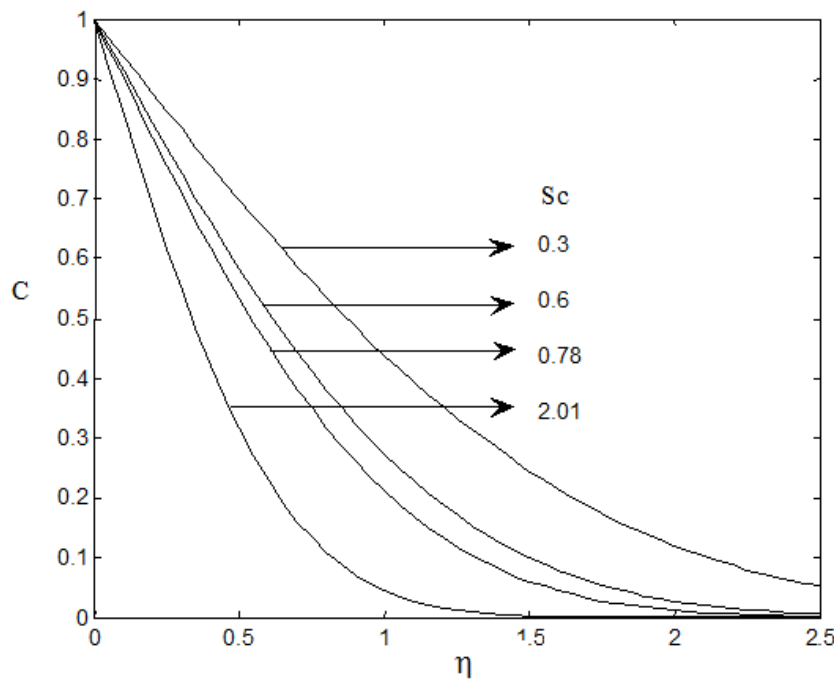

Figure 1: Concentration profiles for different Sc

\section{B. TEMPERATURE PROFILE}

Figure2. shows that the temperature profiles for different values of the thermal radiation parameter $(\mathrm{R}=0.2$, $0.2,2.0,5.0)$ and time $(t=0.2,0.6,0.2,0.2)$. It is observed that the temperature increases with decreasing radiation parameter and the temperature increases with increasing values of the time $t$.

$\theta$

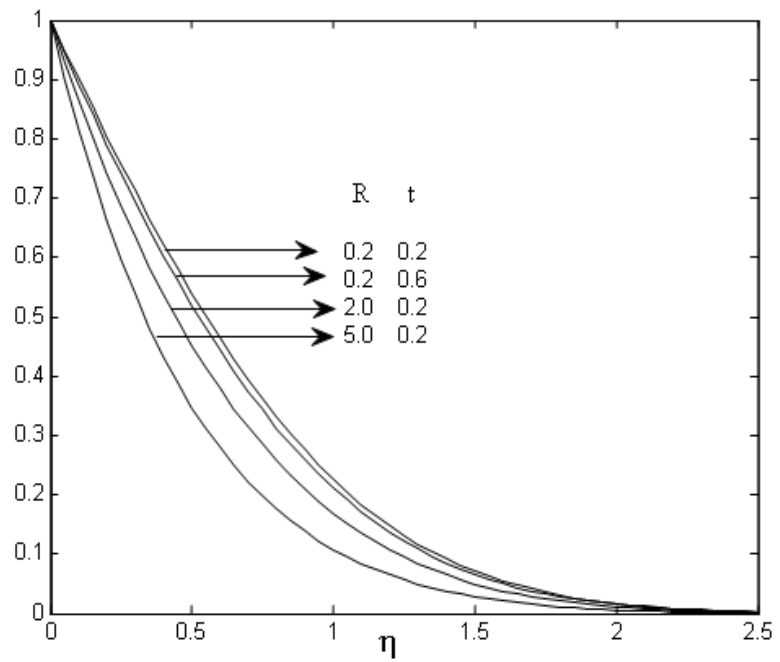

\section{VELOCITY PROFILE}

Figure 3 and 4 shows the variation of primary and secondary velocity profile of $\Omega=\mathrm{M}^{2} \mathrm{~m} /\left(1+\mathrm{m}^{2}\right)$. We observe that the primary velocity $U$ falls when $\Omega$ are increased, but the secondary velocity $\mathrm{V}$ increases with increases in the values of $\Omega$. Figure 5 and 6 present the variation of the primary velocity $U$ and secondary velocity $\mathrm{V}$ profile for different value of Hartmann number $\mathrm{M}$, here the primary velocity increases with decreasing values of $\mathrm{M}$ and the secondary velocity increase with increasing Hartmann number. Figure 7 and 8 , it is noticed that, the primary velocity $\mathrm{U}$ and secondary velocity $\mathrm{V}$ rises due to increasing value of the Hall parameter m. Figure 9 and 10 demonstrate the primary velocity $U$ and secondary velocity $\mathrm{V}$ profile for different value of $\mathrm{R}$, the primary velocity increases with decreasing values of the radiation parameter $\mathrm{R}$, there is rise in the secondary velocity components, due to an increase in the radiation parameter $\mathrm{R}$. The profiles for the primary and secondary velocity are shown in figure 11 and 12 for different value of Gr, Gc. It is observed from these figures that the primary and secondary velocity increases with increasing values of the thermal Grashof number and mass Grashof number. The figure 13 and 14 further show that the primary velocity increases with decreasing values of Schmidt number Sc and the secondary velocity increases with increasing values of Sc. Figure 15 and 16 that the primary and secondary velocity increases with increasing values of $t$.

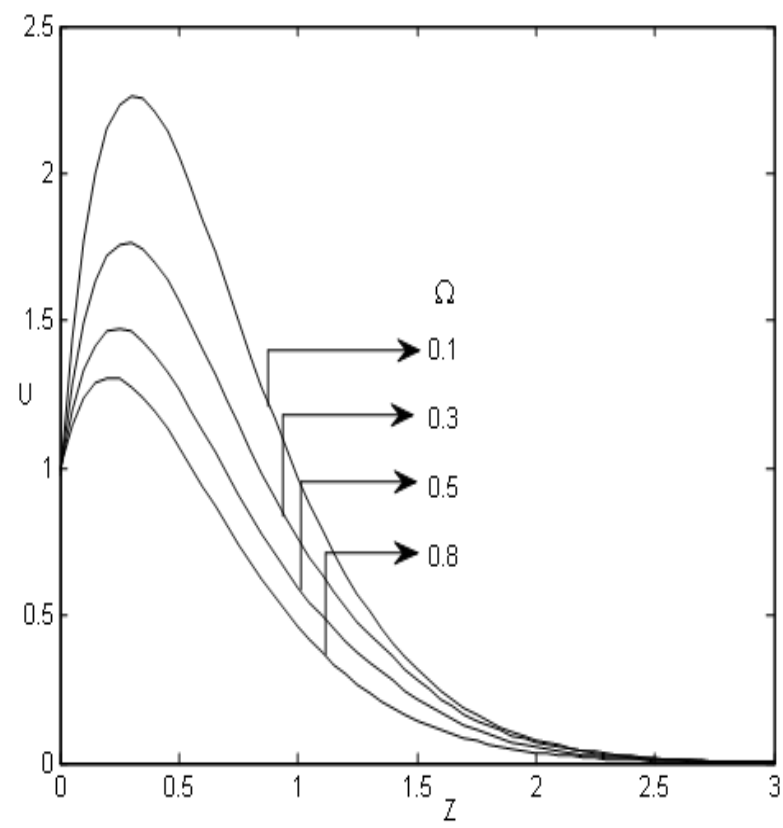

Figure 3: Primary velocity profiles for different $\Omega$

Figure 2: Temperature profiles for different values of $\mathrm{R}$ and $\mathrm{t}$ 


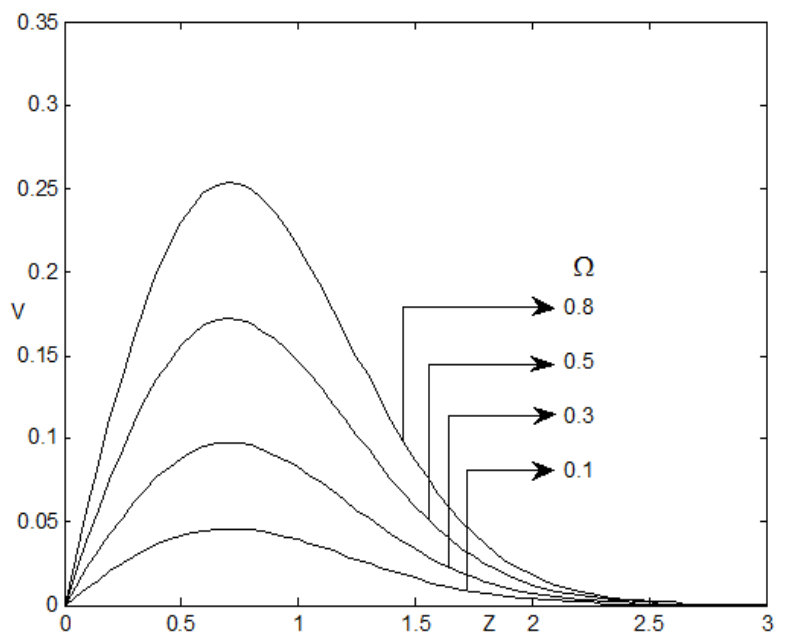

Figure 4: Secondary velocity profiles for different $\Omega$

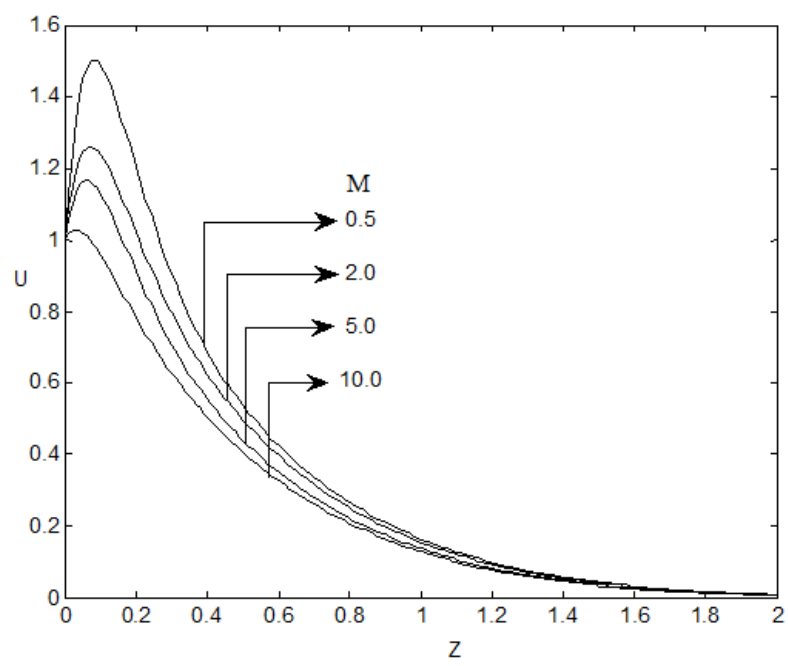

Figure 5: Primary velocity profiles for different $\mathrm{M}$

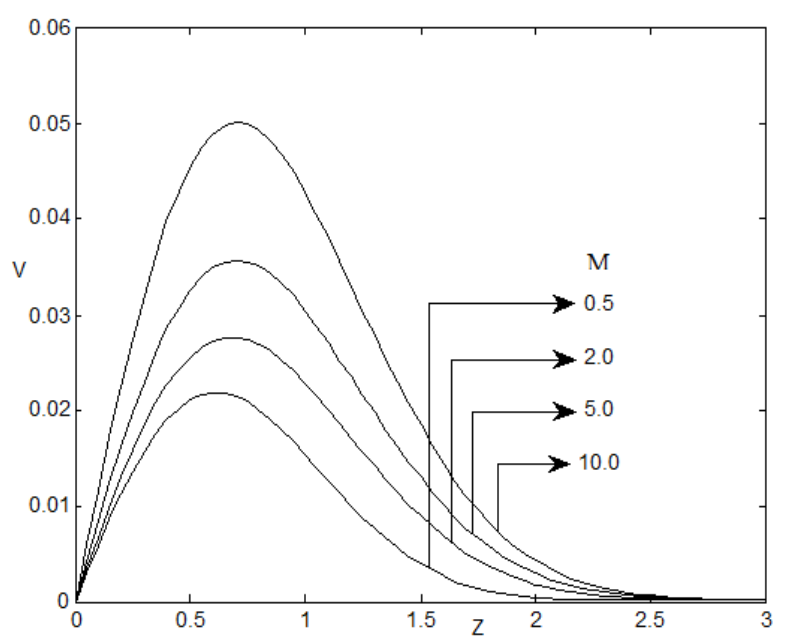

Figure 6: Secondary velocity profiles for different M

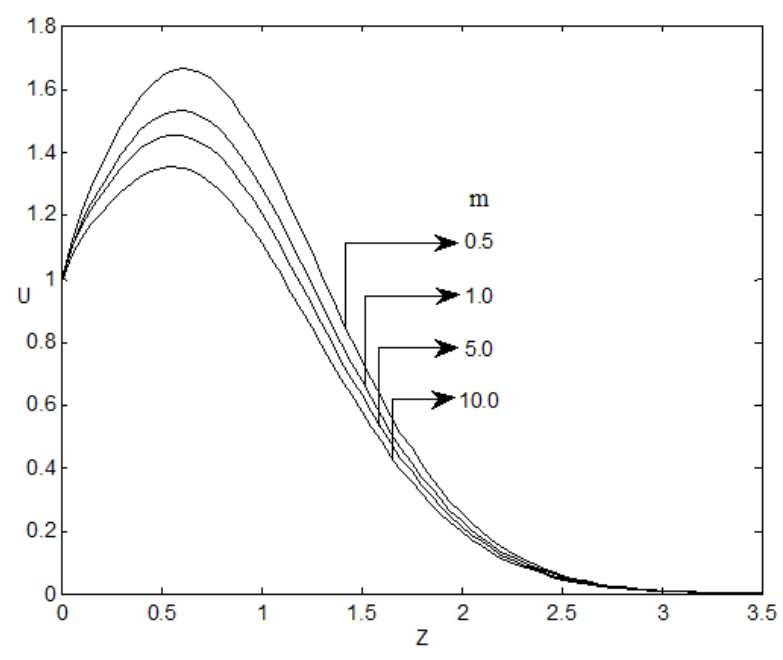

Figure 7: Primary velocity profiles for different $\mathrm{m}$

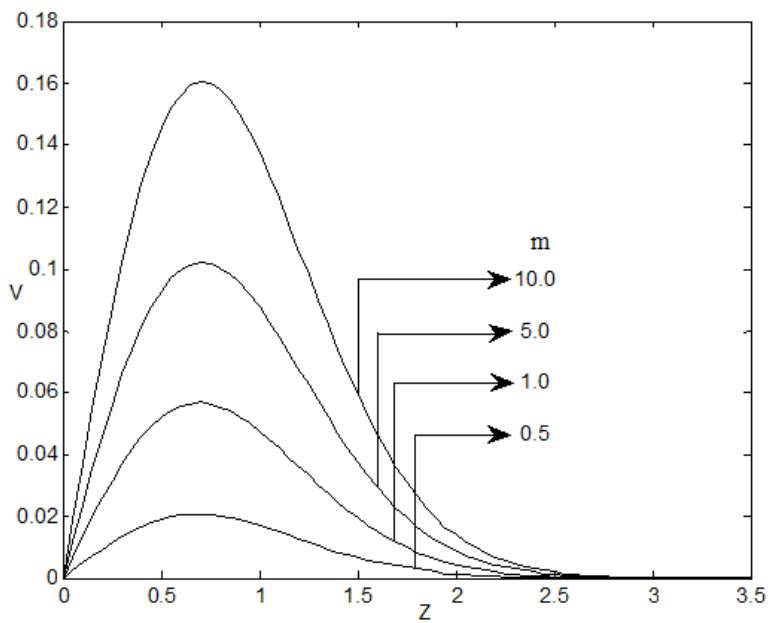

Figure8: Secondary velocity profiles for different $\mathrm{m}$

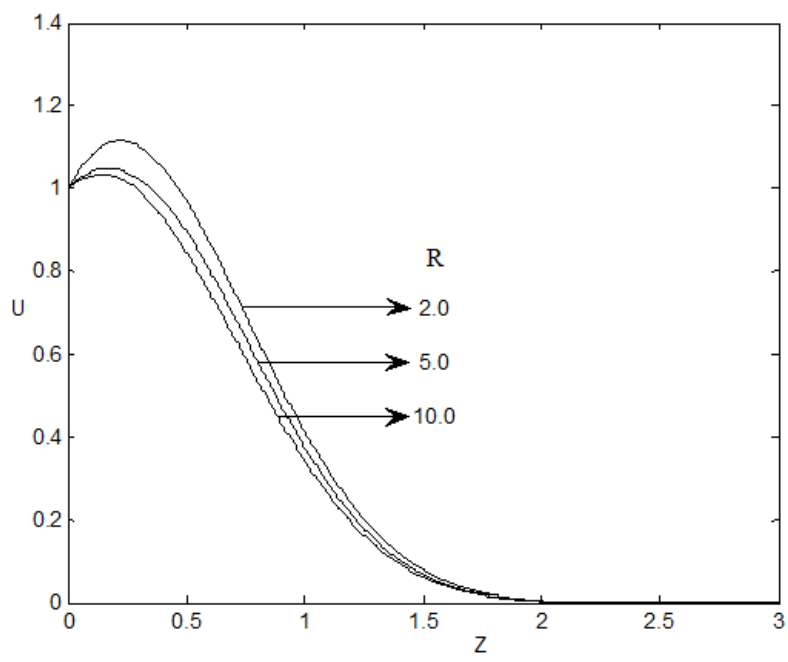

Figure 9: Primary velocity profiles for different $\mathrm{R}$ 


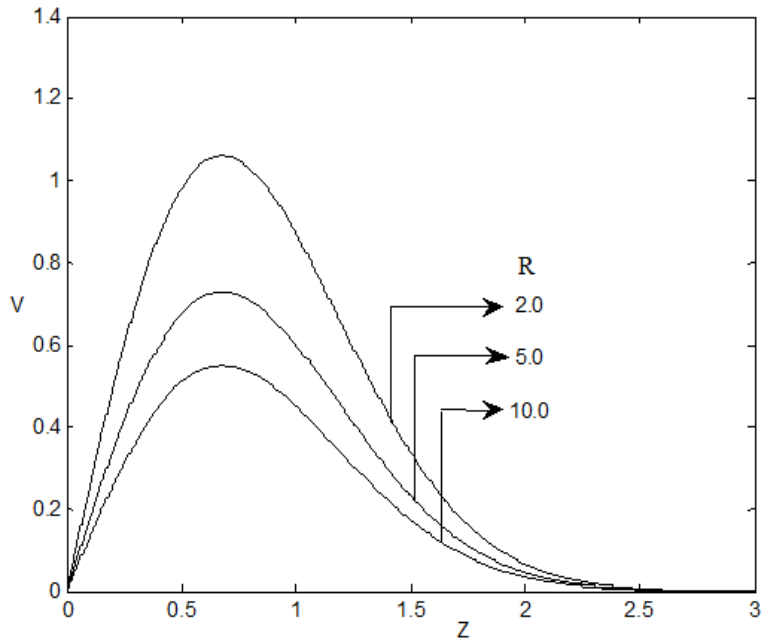

Figure10: Secondary velocity profiles for different $\mathrm{R}$

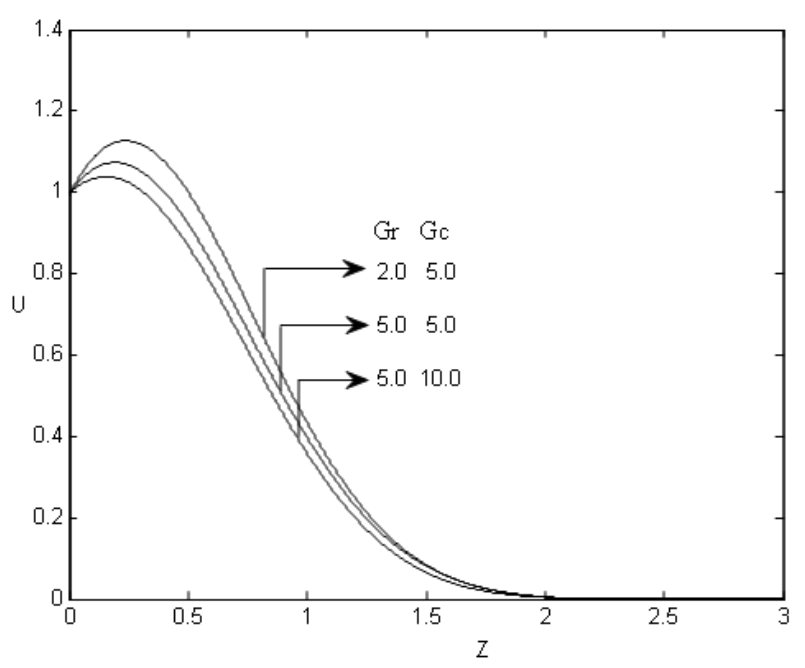

Figure11: Primary velocity profiles for different Gr and Gc

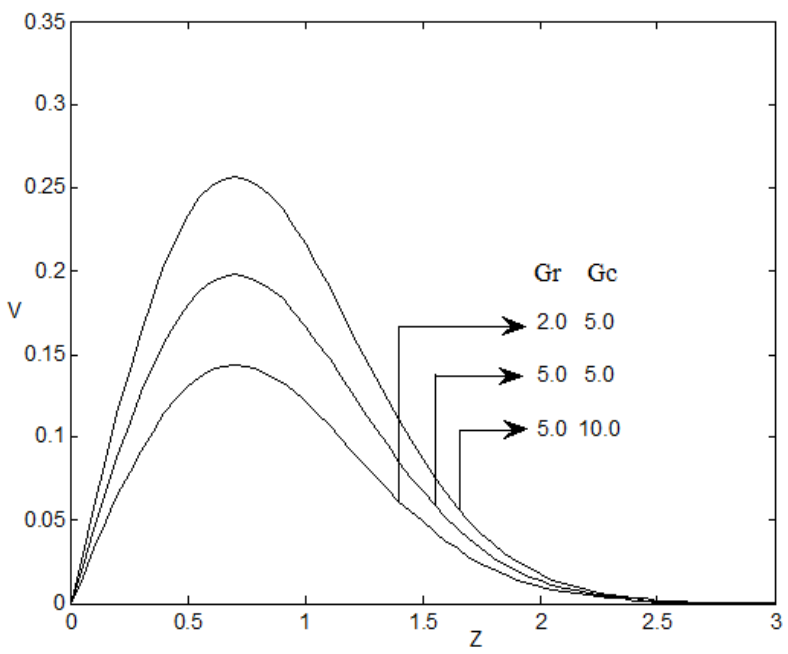

Figure12: Secondary velocity profiles for different Gr and Gc

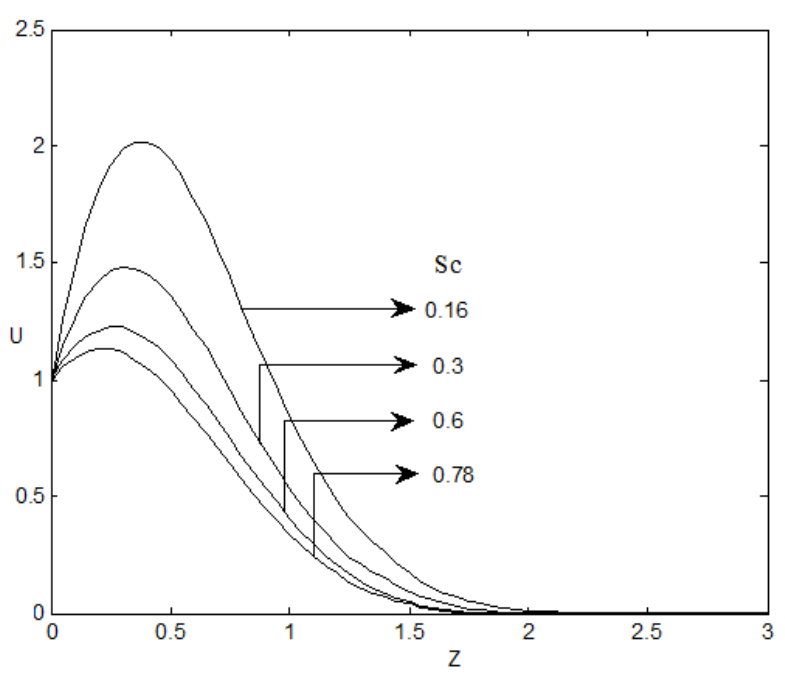

Figure 13: Primary velocity profiles for different Sc

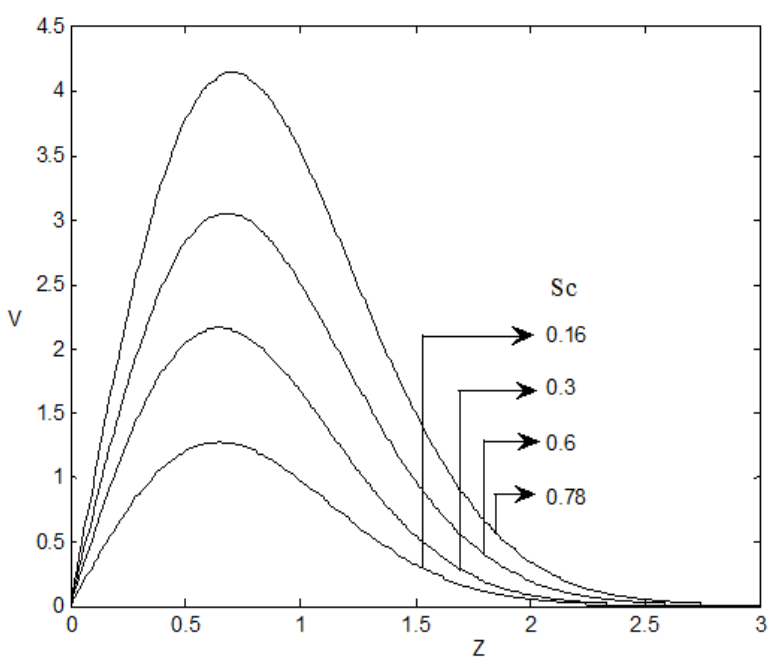

Figure 14: Secondary velocity profiles for different Sc

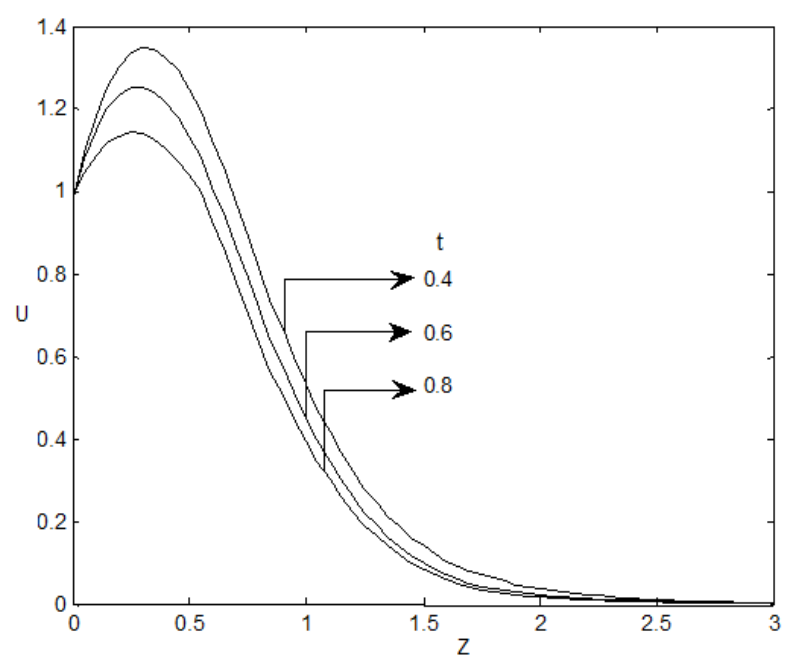

Figure15: Primary velocity profiles for different ' $t$ ' 


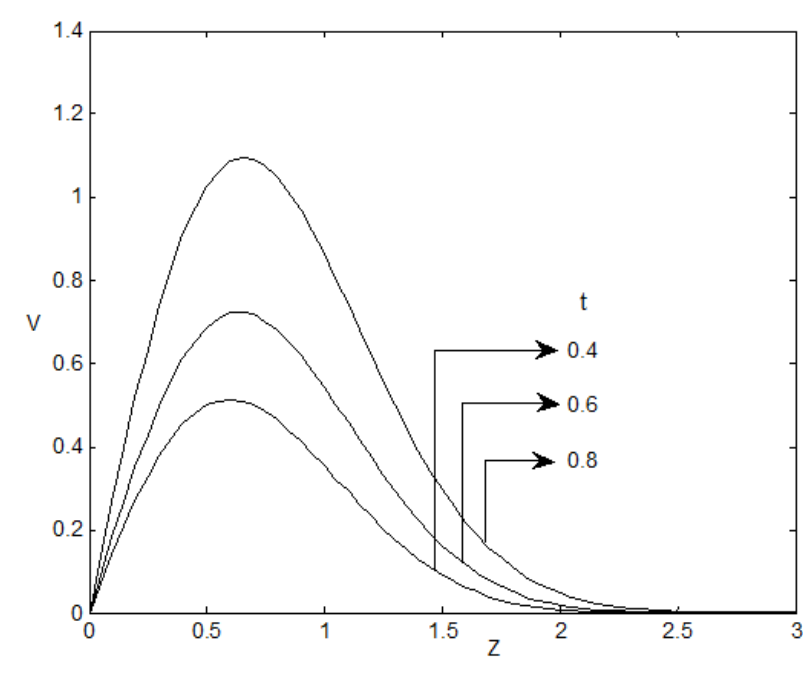

Figure 5.16: Secondary velocity profiles for different ' $t$ '

\section{CONCLUSION}

The paper deals with the unsteady MHD free convection heat and mass transfer flow past an infinite vertical plate. The dimensionless governing equations are solved numerically using Laplace transform method. An analysis on the primary velocity (U) and secondary velocity (V), the following conclusions are listed below:

- $U$ rises due to increasing value of the Hall parameter $(\mathrm{m})$, thermal Grashof number (Gr) and mass Grashof number (Gc), with increasing values of $\mathrm{t}$.

- $\mathrm{U}$ falls when $\Omega$ are increased, the velocity increases with decreasng values of the Hartmann number $(\mathrm{M})$, the velocity increases with decreasing values of the radiation parameter $(\mathrm{R})$, the velocity increases with decreasing values of Schmidt number (Sc).

- $\mathrm{V}$ increases with increasing values of $\Omega, \mathrm{M}, \mathrm{m}$, $\mathrm{R}, \mathrm{Gr}, \mathrm{Gc}$ and $\mathrm{t}$.

- As Schmidt number (Sc) increases the concentration decreases.

- The temperature increases with decreasing radiation parameter, the temperature decrease with increase in the Prandtl number.

\section{REFERENCES}

[1] E.M. Aboeldahabn, Ebarbary, M.E. Elsayed, "Hall current effect on Magneto hydrodynamic free convection flow past a semi infinite vertical plate with mass transfer", Int. J. of Eng. Sci, vol.39, pp.1641-1652, 2001.

[2] M. Acharya, G.C. Dash and L.P Singh, "Hall effect with simultaneous thermal and mass diffusion on unsteady hydro magnetic flow near an accelerated vertical plate," Ind. J. Physics, 75B, pp.68-70, 2001.

[3] Basanth Kumar Jha and Ravindra Prasad, "Free convection and mass transfer effects on the flow past an accelerated vertical plate with heat source", Mechanics Research Communications, vol.17, pp.143-148, 1990.

[4] Chen, "Heat and mass transfer with variable wall temperature and concentration”, Acta Mechanica, 2004

[5] U.N .Das, R.K. Deka and V.M. Soundalgekar, "Radiation effects on flow past an impulsively started vertical infinite plate", Journal of Theoretical Mechanics, vol. 1, pp.111115, 1996.

[6] E.M.A Elbashbeshy, "Heat and mass transfer along a vertical plate with variable surface tension and concentration in the presence of the magnetic field", Int. J. Engg. Sci., vol.34, pp. 515-522, 1997.

[7] R.B.Hetnarski, "An algorithm for generating some inverse Laplace transforms of exponential form", ZAMP26, pp. $249-253,1975$.

[8] P. Ganesh and G. Pilani, "Finite difference analysis of unsteady natural convection MHD past an inclined plate with variable surface heat and mass flux", Int. Journal of Heat and Mass Transfer, vol.47, pp.4449 - 4457, 2004.

[9] N.G. Kafousias and AA.Raptis, "Mass transfer and free convection effects on the flow past an accelerated vertical infinite plate with variable suction or injection", Rev. Roum. Sci. Techn.- Mec, pp.11-22, 1981.

[10] R. Muthucumaraswamy, "The interaction of thermal radiation on vertical oscillating plate with variable temperature and mass diffusion", Theoretical Applied Mechanics, vol. 33, pp.107-121, 2006.

[11] Raptisa, G.J.Tzivanidis and C.P.Peridikis, "Hydro magnetic free convection flow past an accelerated vertical infinite plate with variable suction and heat flux", Letters in heat and mass transfer, vol.8, pp.137-143, 1981.

[12] M.A. Sattar, "Free convection and mass transfer flow through a porous medium past an infinite vertical porous plate with time dependent temperature and concentration", Ind. J. Pure and Appl. Math, vol.23, pp 759-766, 1994.

[13] Takhar (2006). Unsteady flow free convective flow over an infinite vertical porous plate due to the combined effects of thermal and mass diffusion, magnetic field and Hall current. Journal of Heat and Mass Transfer, vol.39, pp.823 -834, 2006. 